\title{
La traducción al español y al francés de los nombres propios en la literatura fantástica: el caso de Juego de Tronos
}

\author{
Translation into Spanish and French of proper names in fantasy \\ literature: analysis of Game of Thrones
}

\author{
Lucila María Pérez Fernández \\ Universidad Europea del Atlántico, España
}

\begin{abstract}
Resumen: El presente artículo compara las estrategias de traducción de los nombres propios en francés y en español de una novela perteneciente al género de literatura fantástica. Para ello se ha utilizado la primera novela de la saga Canción de hielo y fuego, con el fin de establecer una clasificación de los nombres propios, comprobar cuáles son las técnicas de traducción más habituales en ambas lenguas para finalmente compararlas y comprobar en qué casos coinciden.
\end{abstract}

Palabras clave: Juego de Tronos, nombres propios, traducción literaria, técnicas de traducción.

Abstract: This article compares translation techniques for proper names in Spanish and French within a novel considered fantasy literature. For our study we have analyzed the first novel of the saga A song of Ice and Fire with the aim of establishing a classification of proper names, looking into the most common translation techniques in both languages and comparing them in order to find out common patterns.

Keywords: Game of Thrones, proper names, literary translation, translation techniques.

\section{Introdución}

En Las convenciones a la hora de traducir los nombres propios han ido variando a lo largo de los años. Actualmente la tendencia es no traducirlos, pero esto no siempre fue así. En la traducción literaria, y concretamente en la de ficción, los procedimientos pueden variar mucho con respecto a otro tipo de géneros. La razón es que la mayor parte de las veces detrás de cada nombre existe una intención comunicativa del autor, como así lo afirma Lodge (1992: 37) cuando dice "En una novela los nombres nunca son neutrales".

La novela Canción de fuego y hielo contiene una gran variedad de nombres propios, cuya elección, en la mayor parte de los casos, no ha sido casual, sino que presentan connotaciones semánticas que ayudan a definir mejor a los personajes o los lugares o también proporcionan información relevante sobre algún aspecto del hilo argumental.

Por estos motivos, consideramos que la traducción de los nombres propios de la saga ha supuesto todo un desafío para los traductores de las diferentes lenguas y 
mediante el presente artículo pretendemos analizar si los procedimientos de traducción escogidos son los mismos para el español y el francés o averiguar en qué casos difieren y si alguna de las dos lenguas se ha visto obligada a renunciar a transmitir cierta información.

\subsection{Motivación y objetivos}

La elección de la primera novela de la saga Canción de hielo y fuego como corpus obedece a dos razones fundamentales. En primer lugar, a la cantidad de nombres propios (especialmente antropónimos y topónimos) que contiene $y$, en segundo lugar, debido a que una gran parte de esos nombres no desempeñan una mera función designativa, sino que aportan información adicional al lector. Estos motivos son los que han hecho que nos planteemos los siguientes objetivos de investigación:

- Establecer una clasificación de los nombres propios presentes en la primera novela de la saga Canción de hielo y fuego.

- Comprobar cuáles son las técnicas de traducción de los nombres propios más habituales en español y francés.

- Comparar las dos versiones meta y averiguar si los procedimientos de traducción son los mismos en ambas versiones o varían.

\subsection{Metodología}

El corpus objeto de estudio ha sido elaborado a partir de Juego de Tronos, la primera novela de la saga Canción de hielo y fuego del autor George R. R. Martin. Se trata de una obra que se enmarca dentro del género de novela fantástica.

La creación del corpus y su análisis se ha llevado a cabo en varias fases.

Fase 1: Selección de la obra de la cual se extraerán los nombres propios. En este caso se opta por el primer libro de la saga, con el fin de poder de seguir la historia en un orden cronológico.

Fase 2: Extracción de los nombres propios de la obra original inglesa y clasificación.

Fase 3: Extracción de los equivalentes en las obras meta (español y francés)

Fase 4: Análisis de las técnicas de traducción empleadas en cada caso. Para ello seguiremos la clasificación propuesta por Franco (1996).

Fase 5: Extracción de conclusiones

\section{La traducción literaria: el caso de la novela fantástica}

Si cualquier tipo de traducción ya implica un amplio conocimiento lingüístico y cultural por parte del traductor, en el caso de la traducción literaria, la dificultad se ve incrementada por otros factores. La siguiente cita lo explica a la perfección:

El traductor literario, además de enfrentarse a las dificultades que presenta toda traducción, ha de atender a la belleza del texto, a su estilo y sus marcas (lexicales, gramaticales o fonológicas), teniendo en cuenta que las marcas estilísticas en una lengua, pueden no serlo en otra (Ramos 1999: 1)

Por lo tanto, se requiere una precisa comprensión del texto original, lo que según García Yebra (1983: 129), no resulta para nada sencillo pues "la unicidad de la obra 
literaria, su carácter predominantemente subjetivo, la connotación y la plurisignificación que impregnan su estructura verbal, son obstáculos, en parte invencibles, para su comprensión total. Ningún lector puede captar en su totalidad, en todos sus matices, en todas sus vibraciones, el mensaje de una obra literaria".

Como ya indicamos, en el presente artículo nos centraremos en un tipo muy concreto de traducción literaria: el género fantástico. Esta rama presenta unas características propias que determinan las diferentes estrategias de traducción que se van a seguir. En primer lugar, se podría decir que la literatura fantástica se sustenta sobre dos pilares fundamentales. El primero es, sin duda, nuestra realidad, pues en ella encontraremos personas, objetos, lugares con los que también estamos en contacto en nuestro día a día. El segundo es la parte imaginaria o fantástica ideada por el autor y en la que nos podemos encontrar términos o referencias culturales que no existen. Por lo tanto, aquí es cuando deberemos hacer gala de nuestra creatividad y nuestro bagaje para lograr trasladar esos aspectos a la lengua meta de forma que satisfaga al lector final.

\subsection{La saga "Canción de hielo y fuego"}

La obra objeto de estudio en este artículo ha sido Juego de Tronos, la primera de la saga Canción de hielo y fuego, cuyo autor es George Raymond Richard Martin (generalmente conocido como George R. R. Martin). Aunque originalmente el escritor había concebido el libro como una trilogía, su éxito y posterior adaptación a la televisión han propiciado que, de momento, la serie ya cuente con cinco títulos publicados y se espera que lleguen a ser un total de siete. Actualmente la saga ya ha sido traducida a cuarenta y siete idiomas.

La historia se sitúa en un mundo ficticio medieval, en el que encontramos tanto personajes humanos (la mayoría) como otro tipo de seres. Se cree que la principal línea argumental, la guerra dinástica por el control del continente llamado Poniente, está basada en la Guerra de las Dos Rosas, una guerra civil entre dos casas (Lancaster y York) por el trono de Inglaterra. Una de las peculiaridades de esta obra es que cuenta con varios capítulos y en cada uno de ellos se narra la historia desde el punto de vista de un personaje diferente. El éxito de la novela (ya la primera entrega se hizo con el Premio Nebula, uno de los más prestigiosos en el género de la literatura fantástica, en 1996) hizo que la cadena de televisión HBO adquiriera los derechos de la saga en 2008 y en 2011 estrenó la serie que goza incluso de más popularidad que la novela.

\section{La traducción de los nombres propios}

Desde el siglo pasado el estudio de la traducción de los nombres propios ha suscitado un gran interés en el campo de la Traductología y el tipo de estrategias de traducción ha ido variando a lo largo del tiempo. Así, tradicionalmente solían adaptarse los nombres propios de los personajes conocidos (Carlos Marx, Juan Sebastián Bach). Sin embargo, actualmente esa tendencia ha ido desapareciendo en algunos casos y la traducción de los antropónimos se realiza en los casos excepcionales que comentaremos en el siguiente apartado.

Con el caso de los topónimos ocurre algo parecido. En el caso de la literatura, existen diversos procedimientos de traducción en función de si el nombre tiene carga semántica o no y en otro tipo de campos los traductores han ido adoptando 
una serie de criterios comunes que facilitan la labor traductora y contribuyen a garantizar la homogeneidad y coherencia entre textos. A este respecto y como curiosidad podemos recordar la polémica que se formó cuando la traducción automática le jugó una mala pasada a la página web de turismo de Santanderl, en la que Google que se podían observar numerosos errores de traducción al inglés (por ejemplo, el Centro Botín había sido traducido literalmente como "Loot Center", lo que equivale a "centro del saqueo") y también cuando tradujo a español un gran número de calles catalanas² (Mare de Déu del Coll pasó a ser Virgen del Cuello, la avenida Pau Casals se rebautizó como Pablo Casals y la calle Sostres se convirtió en calle Techos). La trascendencia de este incidente se vio también motivada por cuestiones de origen ideológico, pues como indica Fundéu los topónimos no son inocentes, por lo que deberemos ser también conscientes de que optar por un nombre $u$ otro puede tener una connotación política, esta misma razón es la que llevó a Fundéu ${ }^{3}$ a recomendar el uso de los nombres A Coruña, Girona, Lleida y Ourense.

Por lo tanto, la labor no es fácil y ante la duda, lo más recomendable es recurrir a fuentes documentales, tales como atlas, enciclopedias o listas de topónimos.

\title{
3.1. Estrategias de traducción para los nombres propios
}

\subsubsection{Los antropónimos}

De acuerdo con Cuéllar (2014: 364), la presencia o ausencia de connotación será lo que determine si un antropónimo se va a traducir o no. Por lo general, si el nombre no contiene ningún tipo de connotación o significado, lo habitual es transferirlo, es decir, se mantiene igual que en la obra original. Por el contrario, cuando el nombre propio tiene un matiz que ayuda al lector a conocer mejor al personaje (por ejemplo, si el nombre hace referencia al aspecto físico del personaje, a su lugar de procedencia o aporta cualquier otro tipo de información), entonces dichas connotaciones se intentarán preservar en la lengua meta. A este respecto Newmark (1988: 215) hace la siguiente propuesta:

\begin{abstract}
Where both connotations (rendered through sound effects and/or transparent names) and nationality are significant, I have suggested that the best method is first to translate the word that underlies the SL proper name into the $\mathrm{TL}$, and then to naturalise the translated word back into a new SL proper name - but normally only when the character's name is not yet current amongst an educated TL readership.
\end{abstract}

Cabe aquí señalar que en inglés el uso de nombres propios con un determinado matiz o rasgo definitorio de un personaje es mucho más habitual que en español, de ahí que muchas veces veamos que este tipo de nombres acaban manteniéndose igual en la versión en lengua meta.

Asimismo, traduciremos los nombres de monarcas, príncipes, emperadores o papas.

\footnotetext{
1 https://www.hoy.es/sociedad/traductor-google-juega-20180118201046-nt.html

2 https://www.lavanguardia.com/vida/20111216/54241176993/google-cambia-nombre-calles-

catalunya.html

${ }^{3}$ http://www.fundeu.es/noticia/traducir-o-no-los-toponimos-3617/
} 


\subsubsection{Los topónimos}

Ainaud, Espunya y Pujol (2003: 229) explican que, aunque no hay normas estrictas en lo que concierne a la traducción de topónimos, sí que existen una serie de procedimientos habituales que muchos traductores suelen aplicar.

La regla general es que los topónimos no se traducen, pero, como toda regla, esta también cuenta con excepciones. Las que recogen Ainaud, Espunya y Pujol (2003: 229) son las siguientes:

Así, por lo general, se traducen:

- los nombres de los continentes y de los topónimos que los incluyen (South America > América del Sur)

- los nombres de mares y océanos (the Red Sea > el mar Rojo),

- los topónimos compuestos de un nombre común y adjetivo o complemento (Rocky Mountains > montañas Rocosas),

- los topónimos compuestos de adjetivo o complemento y nombre propio (New York > Nueva York),

- los topónimos que son traducciones o adaptaciones de otras lenguas (Sweden > Suecia),

- los topónimos con forma en castellano consolidada (Thames > Támesis).

\section{1.3. Otros nombres propios}

Recogemos en este apartado algunos de los ejemplos recogidos por Moya y Ainaud, Espunya y Pujol (2003). La lista no pretende ni mucho menos ser exhaustiva, pero sí que nos sirve para fijarnos en otro tipo de nombres que también pueden requerir el uso de variadas técnicas de traducción.

Por un lado, es habitual traducir los nombres de los partidos políticos, los nombres de organismos internacionales que disponen de nombres multilingües y los nombres de los ministerios cuando son lo suficientemente descriptivos (Newmark, 1992: 140-2), por ejemplo, Ministry of Defence > Ministerio de Defensa. También se traducen los nombres de ejércitos y compañías y los de las operaciones bélicas (Moya 1993:245). Por último, en el caso de los nombres de monedas, la práctica más común es adaptarlos al español (dólar, libra).

Cabe también aquí destacar que cuando encontramos en un texto referencias a nombres de festividades del calendario religioso, emplearemos el equivalente correspondiente en la lengua meta (Easter > Pascua), pero si se trata de celebraciones más lejanas a la cultura de llegada es posible que las estrategias de traducción varíen. Así, Ainaud, Espunya y Puyol (2003: 233) indican que emplearemos el calco con las festividades que son más conocidas como Thanksgiving Day > Día de Acción de Gracias o Fourth of July > Cuatro de julio. Sin embargo, en otros casos necesitaremos añadir algún tipo de información. Dichos autores ponen como ejemplo el caso de Labor Day, pues si lo traducimos usando el calco "Día del Trabajo", podríamos inducir al lector a pensar que ambas fiestas se corresponden en fecha cuando en realidad no es así. 
Por otro lado, Ainaud, Espunya y Pujol (2003: 234) señalan que existen otro tipo de nombres propios que generalmente no se traducen, por ejemplo, los nombres de empresas o marcas comerciales registradas (a no ser que el producto se comercialice con otro nombre en el país meta, ej: Vauxhall > Opel).

\subsection{Técnicas de traducción de nombres propios}

Franco (1996) distingue doce tipos de técnicas de traducción de nombres propios desde una perspectiva cultural y las divide en dos grandes estrategias: conservación y sustitución.

\begin{tabular}{|l|l|}
\hline I. Conservación & II. Sustitución \\
\hline 1. Repetición & 7. Universalización limitada \\
2. Adaptación ortográfica & 8. Universalización absoluta \\
3. Adaptación terminológica & 9. Naturalización \\
4. Traducción lingüística & 10. Adaptación ideológica \\
5. Glosa extratextual & 11. Omisión \\
6. Glosa intratextual & 12. Creación autónoma \\
\hline
\end{tabular}

1. Repetición: reproducir la grafía original del nombre propio. Ej.: Arthur $>$ Arthur

2. Adaptación ortográfica: realizar leves cambios en la grafía original. Ej.: bin Laden > ben Laden.

3. Adaptación terminológica: transformación formal del nombre propio cuando este cuenta con una versión oficial distinta en lengua meta. Ej.: London > Londres.

4. Traducción lingüística: transferencia parcial o total del contenido semántico del significante común (adjetivos y apelativos principalmente) que compongan el nombre propio en la que el resultado sigue percibiéndose como un elemento de la cultura original. Ej.: New York > Nueva York.

5. Glosa extratextual: uso de cualquiera de las cuatro anteriores estrategias acompañándolas de una explicación del traductor.

6. Glosa intratextual: incluir una explicación integrada dentro del texto meta. Ej.: Metropole > hotel Metropole.

7. Universalización limitada: sustitución del nombre propio original por un referente distingo, pero que todavía se pueda seguir considerando exótico. Ej: Mart > Martin

8. Universalización absoluta: neutralización completa del nombre original. Ej.; Nietzsche > un filósofo.

9. Naturalización: sustitución del nombre propio por otro cuyo referente se considere específico de la cultura meta. Ej.: Harry > Antonio.

10. Adaptación ideológica: cambio de una formulación original que se considera ideológicamente inaceptable por otra que encaje mejor en los valores de la cultura meta. Ej.: God and Clod > Ángel y bestia. 
11. Omisión: ocurre cuando se suprimen detalles que se consideran innecesarios o cuando se condensan. Ej.: United States Senate > Senado

12. Creación autónoma: añadir un nombre propio donde no existía nada en el texto original. Ej.: many grew into cities, such as York, England. > muchos llegaban a ciudades, como York en Inglaterra o León en España.

\section{Análisis y resultados}

Una vez compilado el corpus, clasificamos todos los nombres propios estableciendo para ello diversas categorías que nos faciliten el posterior análisis. Así, finalmente los ejemplos que comentaremos se han agrupado en torno a ocho categorías. Con el fin de facilitar la lectura del análisis al lector presentaremos los datos en formato de tabla.

\subsection{Los antropónimos}

Si hay algo que caracterice a la obra objeto de estudio en el presente artículo son los nombres propios. Solo en la primera novela encontramos 335 nombres de personas $y$, tanto en español como en francés, la mayoría de ellos se mantienen igual que en el original, es decir, a la hora de trasvasarlos a las lenguas metas se ha optado por transcribirlos. Esta decisión se debe, probablemente, a que muchos de esos nombres no tienen ningún tipo de connotación semántica (Robert Baratheon, Cersei Lannister). No obstante, como podemos ver en la siguiente tabla, esta regla no se cumple para todos los casos:

\begin{tabular}{|l|l|l|}
\hline INGLÉS & ESPAÑOL & FRANCÉS \\
\hline Robert Baratheon & Robert Baratheon & Robert Baratheon \\
\hline Cersei Lannister & Cersei Lannister & Cersei Lannister \\
\hline Theon Greyjoy & Theon Greyjoy & Theon Greyjoy \\
\hline Gerold Hightower & Gerold Hightower & Gerold Hightower \\
\hline Cotter Pyke & Cotter Pyke & Cotter Pyke \\
\hline
\end{tabular}

Si nos fijamos en los apellidos Greyjoy y Hightower vemos que literalmente podrían traducirse como "alegría gris" y "torre alta", respectivamente. Sin embargo, en las dos versiones meta analizadas ambos apellidos se han mantenido igual que en el texto original. Recientemente en una entrevista ${ }^{4}$, la traductora de la novela admitía que la traducción (o no traducción) del apellido Hightower había sido motivo de discusión entre el equipo, pero finalmente se había llegado a esa decisión, al considerar que este no tenía significado alguno dentro de la acción.

Mención aparte merecen los casos en los que ambas lenguas meta realizan una transcripción de los nombres, pero en el caso concreto del francés se adaptan a la grafía del idioma, como se muestra en la siguiente tabla:

\footnotetext{
${ }^{4}$ https://www.youtube.com/watch?v=EOq9Uo WOCQ
} 


\begin{tabular}{|l|l|l|}
\hline INGLÉS & ESPAÑOL & FRANCÉS \\
\hline Ser Theo & Ser Theo & Ser Théo \\
\hline Lord Beric Dondarrion & Lord Beric Dondarrion & Lord Béric Dondarrion \\
\hline
\end{tabular}

También observamos casos en los que el nombre se mantiene igual en una de las lenguas y en la otra varía:

\begin{tabular}{|l|l|l|}
\hline INGLÉS & ESPAÑOL & FRANCÉS \\
\hline Jon Snow & Jon Nieve & Jon Snow \\
\hline Mya Stone & Mya Piedra & Mya Stone \\
\hline Martyn Rivers & Martyn Ríos & Martyn Rivers \\
\hline
\end{tabular}

Los apellidos Snow, Stone y Rivers tienen una razón de ser en la obra, pues los tres se utilizan en el caso de hijos bastardos, el primero para los del Norte, el segundo para los del Valle y el tercero para los hijos bastardos de las tierras de los ríos. Como se muestra en la tabla, las estrategias de traducción difieren en ambas lenguas, pues mientras que en español se ha optado por traducirlos, en francés todos ellos se mantienen igual que en inglés.

En otros casos, vemos que ocurre justo lo contrario y mientras que en español se recurre a la transcripción en apellidos como Oakheart (literalmente "corazón de roble"), Hornwood ("cuerno de madera"), Umber ("marrón oscuro") o Blackwood ("madera negra"), el francés opta por mantener parte del significado implícito en el nombre, aunque en muchos casos no podemos realmente hablar de traducción literal.

\begin{tabular}{|l|l|l|}
\hline INGLÉS & ESPAÑOL & FRANCÉS \\
\hline Ser Addam Marbrand & Ser Addam Marbrand & Ser Addam Marpheux \\
\hline Ser Arys Oakheart & Ser Arys Oakheart & Ser Arys du Rouvre \\
\hline Lord Hornwood & Lord Hornwood & Lord Corbois \\
\hline Lord Umber & Lord Umber & Lord Omble \\
\hline Lord Blackwood & Lord Blackwood & Lord Nerbosc \\
\hline
\end{tabular}

No obstante, también encontramos ejemplos en los que ambas lenguas meta han optado por la traducción literal.

\begin{tabular}{|l|l|l|}
\hline INGLÉS & ESPAÑOL & FRANCÉS \\
\hline Ser Garth Greenfield & Ser Garth Pradoverde & Ser Garth Verchamps \\
\hline Ser Hugh of the Vale & Ser Hugh de Valle & Ser Hugh du Val \\
\hline
\end{tabular}

En el siguiente ejemplo se puede observar la similitud de las técnicas empleadas entre los dos pares de lenguas meta para la traducción de los antropónimos. Aunque en este caso el español tiende un poco más a la repetición y el francés más 
a la traducción lingüística, vemos que los resultados son bastante parecidos. Se observa, además, un uso más elevado de la adaptación ortográfica en el caso del francés.

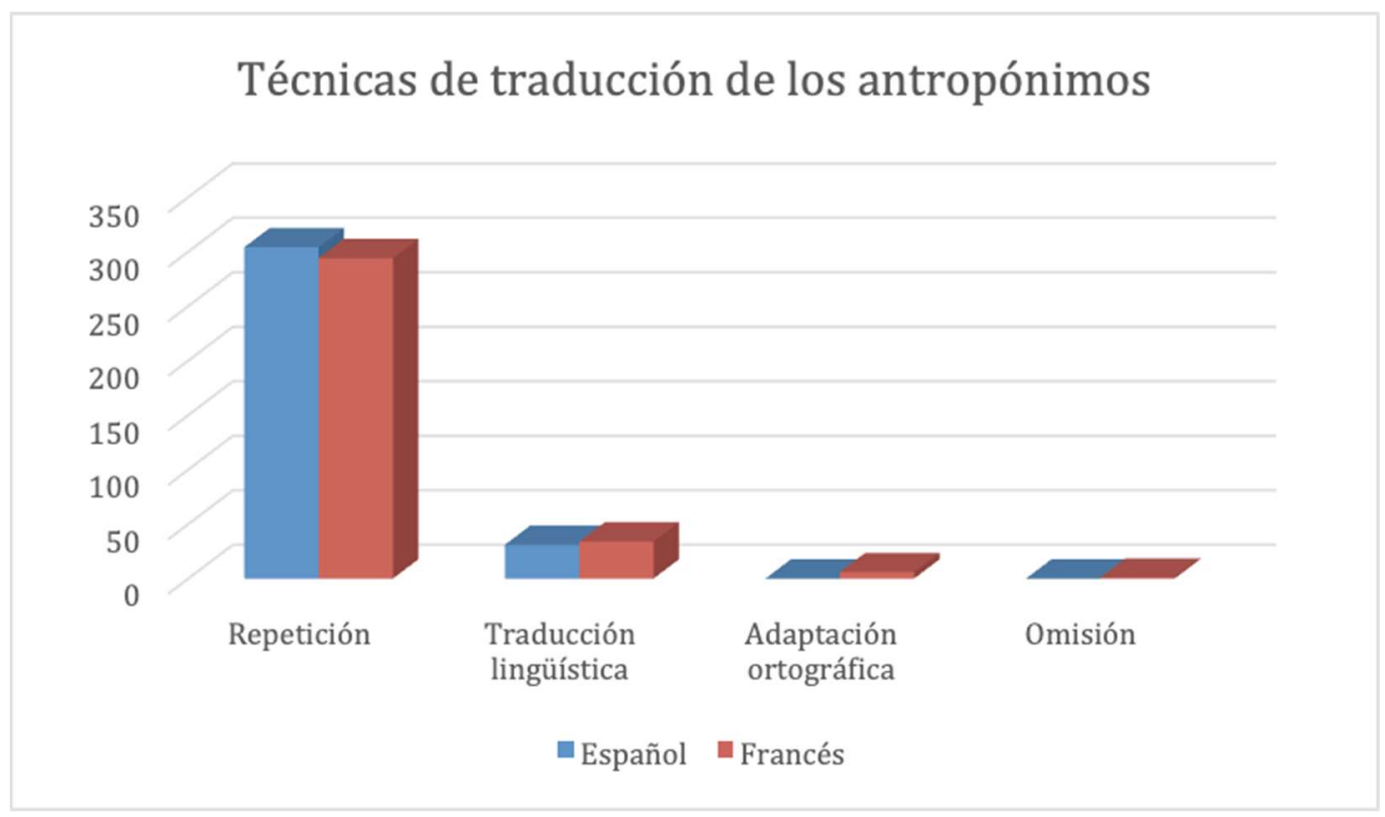

\subsection{Los sobrenombres}

En la saga en general se observa que muchos de los personajes cuentan con un sobrenombre que, en la mayor parte de los casos, se han trasvasado a las lenguas meta mediante traducción literal.

\begin{tabular}{|l|l|l|}
\hline INGLÉS & ESPAÑOL & FRANCÉS \\
\hline Brandon the Builder & Branden el Constructor & Brandon le Bâtisseur \\
\hline The wildlings & Los salvajes & Les sauvageons \\
\hline Three-Finger Hobb & Hobb Tresdedos & Hobb Trois-Doigts \\
\hline $\begin{array}{l}\text { Merchant Captain Byan } \\
\text { Votyris }\end{array}$ & $\begin{array}{l}\text { El capitán mercante Byan } \\
\text { Votyris }\end{array}$ & $\begin{array}{l}\text { Le capitaine-marchand Byan } \\
\text { Votyris }\end{array}$ \\
\hline The Usurper & El Usurpador & L'Usurpateur \\
\hline The Kingslayer & El Matarreyes & Le Régicide \\
\hline $\begin{array}{l}\text { Howland Reed, the Little } \\
\text { crannogman }\end{array}$ & $\begin{array}{l}\text { Howland Reed, el menudo } \\
\text { lacustre }\end{array}$ & $\begin{array}{l}\text { Le petit échanson Howland } \\
\text { Reed }\end{array}$ \\
\hline The Hound & El Perro & Le Limier \\
\hline The dwarf & El gnomo & Le lutin \\
\hline
\end{tabular}

De la lista anterior merece una mención especial el caso del sobrenombre "the Little crannogman" y sus traducciones a las lenguas meta analizadas. En primer lugar, debemos explicar que el término crannog hace referencia a un tipo de construcción irlandesa típica de la Edad Media y de la Prehistoria que se levantaba 
sobre el agua (generalmente lagos o ríos). Por lo tanto este mote sería literalmente "el pequeño hombre crannog". Para su trasvase a español se ha utilizado el adjetivo "lacustre" que el Diccionario de la Real Academia Española define como sigue:

\section{Lacustre}

1. adj. Perteneciente o relativo a los lagos.

2. adj. Que habita, está o se realiza en un lago o en sus orillas.

El caso del francés llama un poco más la atención, pues se ha empleado el término "échanson", que hace referencia a la persona que se ocupaba de servir las bebidas en la mesa de la corte real y que en español solemos conocer como "copero".

También cabe destacar el caso del sobrenombre de Tyrion Lannister. En inglés, se emplea el término "dwarf" (literalmente "enano"). Sin embargo en español el libro habla del "gnomo" y en francés se emplea el término "lutin" (literalmente "duende"). Resulta llamativo que en la serie doblada al español sí se refieran a este personaje como "enano". No obstante, como ya indicamos en el presente estudio tan solo nos estamos centrando en la primera entrega de la saga, por lo que habría que analizar el resto para saber si el término "gnomo" se mantiene o no.

Por último, uno de los sobrenombres que presenta una traducción en español, pero que en francés se ha mantenido igual que en la lengua original es el del personaje Petyr Baelish, conocido como "Littlefinger". En la versión meta española, este mote se ha traducido literalmente, sin embargo en francés se ha mantenido en inglés, a pesar de que el término cuenta con la traducción "auriculaire".

\begin{tabular}{|l|l|l|}
\hline INGLÉS & ESPAÑOL & FRANCÉS \\
\hline Littlefinger & Meñique & Littlefinger \\
\hline
\end{tabular}

\subsection{Los topónimos y lugares}

La mayoría de los topónimos presentes en la obra aportan algún tipo de información sobre el lugar. En algunos casos se refieren a la apariencia (Red Keep, Castle Black) y en otros están relacionados con las diferentes Casas que protagonizan la historia. Así, Dragonstone es la tierra de los Targaryen, cuyo emblema es el dragón, o The Eyrie es el asentamiento principal de los Arryn, en cuyo emblema encontramos una luna creciente y un halcón.

Observamos aquí que para adaptar los topónimos a las dos lenguas meta hay una clara tendencia a la conservación y dentro de esta a la traducción lingüística.

\begin{tabular}{|l|l|l|}
\hline INGLÉS & ESPAÑOL & FRANCÉS \\
\hline The Wall & El Muro & Le Mur \\
\hline King's landing & Desembarco del Rey & Port-Réal \\
\hline The haunted forest & El bosque Encantado & La forêt hantée \\
\hline The Isle of Faces & La Isla de los Rostros & L'lle-aux-Faces \\
\hline The Red Keep & La Fortaleza Roja & Le Donjon Rouge \\
\hline
\end{tabular}




\begin{tabular}{|l|l|l|}
\hline The King's Gate & La Puerta del Rey & La porte du Roi \\
\hline Horn Hill & Colina Cuerno & Corcolline \\
\hline The Lands of the Long Summer & Las Tierras del Largo Verano & Les Pays du Grand Eté \\
\hline The Iron Islands & Las Islas del Hierro & Les îles de Fer \\
\hline Dragonstone & Rocadragón & Peyredragon \\
\hline Castle Black & El Castillo Negro & Châteaunoir \\
\hline
\end{tabular}

Una de las que más quebraderos de cabeza supuso fue King's Landing, pues se trata de una referencia a cuando el primer Targaryen llegó montado en su dragón. En español la versión traducida opta por el verbo "desembarcar", que significa "llegar a un lugar", mientras que en francés se ha modificado ligeramente el sentido al hablar de "Port-Réal", que literalmente quiere decir "Puerto Real". El corazón de Desembarco del Rey es la Fortaleza Roja (The Red Keep). En inglés el término "keep" hace referencia a la torre principal de un castillo, al igual que el término "donjon", que fue el escogido para la versión meta francesa. La versión española optó por "fortaleza", un término que, en cierto modo, podríamos también considerar un equivalente exacto, puesto que hace referencia a cualquier recinto fortificado, como un castillo o una ciudadela.

En el caso de Dragonstone, vemos que en ambas lenguas meta se ha efectuado la traducción literal, con la peculiaridad de que en la versión francesa se ha recurrido al uso del término "peyre" que en occitano significa "piedra".

Asimismo, constatamos casos en los que una lengua meta opta por traducir el topónimo, mientras que la otra lo mantiene igual que en la lengua original. Algunos de los ejemplos aparecen recogidos en la siguiente tabla:

\begin{tabular}{|l|l|l|}
\hline INGLÉS & ESPAÑOL & FRANCÉS \\
\hline Winterfell & Invernalia & Winterfell \\
\hline Moat Cailin & Foso Cailin & Moat Cailin \\
\hline Lannisport & Lannisport & Port-Lannis \\
\hline Karhold & Bastión Kar & Karhold \\
\hline Raventree & El Árbol de los Cuervos & Raventree \\
\hline Valyria & Valyria & La Valyrie \\
\hline The Eyrie & El Nido de Águilas & Eyrié \\
\hline Volantis & Volantis & Volantys \\
\hline
\end{tabular}

Uno de los topónimos que más protagonismo adopta a lo largo de la obra analizada es Winterfell, considerada la capital del Norte, donde reside la familia Stark. Probablemente sería uno de los que más preocupación generaría a cualquier traductor. Los de nuestras versiones meta han optado por técnicas de traducción diferentes, mientras que en francés se ha mantenido la versión en inglés, en español se ha logrado hallar un equivalente que mantiene la idea de "Winter" y a la vez 
añade el sufijo griego - alia para hacer que el término encaje a la perfección en el contexto medieval en el que se enmarca la historia.

En otros casos vemos que, con el fin de proporcionar toda la información al lector, el español opta por traducir los topónimos literalmente. Así, "Raventree" pasa a ser "El Árbol de los Cuervos" o "The Eyrie" se traduce como "El Nido de Águilas". Sin embargo, en ambos casos la versión francesa se mantiene coherente con su decisión de no traducir los topónimos. Tan solo hallamos dos casos en los que la versión meta francesa presenta unas ligeras variaciones (tan solo ortográficas), con respecto al texto original. Estos son "Valyria", que en francés pasa a ser "La Valyrie" y "Volantis", que se adapta cambiando la "i" por la "y" (Volantys).

Por lo tanto, en el caso de los topónimos de nuevo observamos una alta tendencia a la traducción lingüística para los topónimos que poseen algún tipo de carga semántica y a la repetición, especialmente para traducir los nombres de los lugares extranjeros (Tyrosh, Braavos, Myr, Qohor). Comprobamos, además, que el francés recurre a una mayor variedad de técnicas, aunque su uso resulta meramente anecdótico, tal y como muestra el siguiente gráfico.

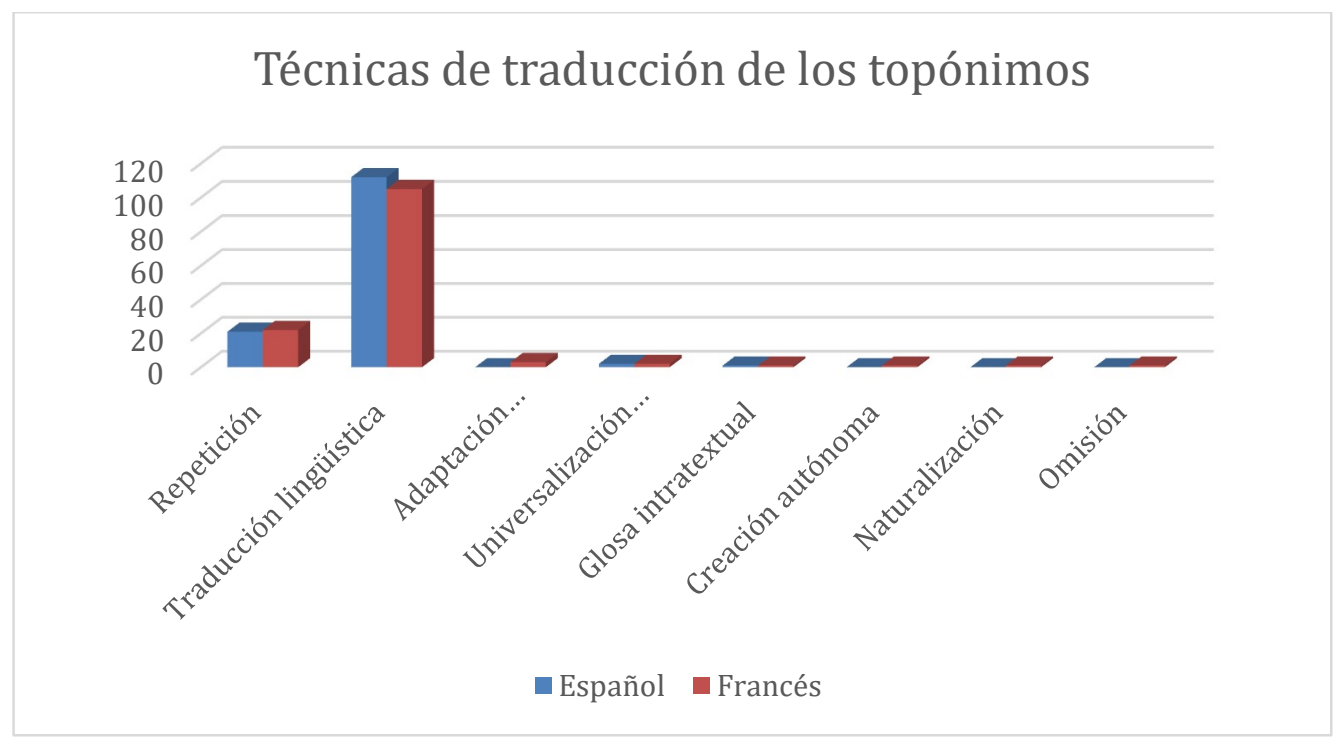

\subsection{Nombres de mares, ríos, arroyos y lagos}

En la novela Juego de Tronos aparecen una gran cantidad de nombres de mares, ríos, arroyos y lagos. Como se puede observar en la tabla, la mayoría de ellos se han traducido literalmente en ambas versiones. No obstante, también se encuentran algunos casos en los que se ha optado por una traducción más creativa.

Riverrun es la fortaleza ancestral de la Casa Tully, situada en la punta donde confluyen los ríos Forca Roja y Piedra Caída, de ahí su nombre original. La traducción literal de dicho término sería "caudal/corriente del rio", una idea que se aproxima más a la de la versión francesa "Vivesaigues", que literalmente significa aguas (traducido del occitano "aigue") vivas. En español se ha optado por el término "Aguasdulces" que, sin ser tan literal, sigue recordando igualmente a un río, pero no presenta exactamente el mismo matiz que en inglés. 
En el caso de "The Womb of the World" observamos un cambio de punto de vista en las dos versiones meta. El término inglés "womb" (literalmente "útero") pasa a ser "vientre" en la versión española y "nombril" (ombligo) en la versión francesa.

\begin{tabular}{|l|l|l|}
\hline INGLÉS & ESPAÑOL & FRANCÉS \\
\hline Riverrun & Aguasdulces & Vivesaigues \\
\hline The narrow sea & El mar Angosto & Le bras de mer \\
\hline The Jade Sea & El mar Jade & La mer de Jade \\
\hline The Trident & El Tridente & Le Trident \\
\hline The Summer Sea & El mar del Verano & La mer d'Eté \\
\hline The Bite & El Mordisco & La Morsure \\
\hline The Blackwater Rush & El Aguasnegras & La Néra \\
\hline Alyssa's Tears & Lágrimas de Alyssa & Les Larmes d'Alyssa \\
\hline The Womb of the World & Vientre del Mundo & Le Nombril du Monde \\
\hline The Green Fork & El Forca Verde & La Verfurque \\
\hline The Red Fork & El Forca Roja & La Ruffurque \\
\hline The Tumblestone & El Piedra Caída & La Culbute \\
\hline The Blue Fork & El Forca Azul & La Bleufurque \\
\hline Gods Eye & Ojo de Dioses & L'Oeildieu \\
\hline
\end{tabular}

\subsection{Nombres de periodos de tiempo}

Los periodos de tiempo que encontramos en esta primera entrega de la saga se han traducido literalmente en ambas versiones meta, como así se puede observar en la tabla:

\begin{tabular}{|l|l|l|}
\hline INGLÉS & ESPAÑOL & FRANCÉS \\
\hline The Age of Heroes & La Edad de los Héroes & L'Age des Héros \\
\hline The Long Night & La Larga Noche & la Longue Nuit \\
\hline The Dawn Age & La Era del Amanecer & L'Age de l'Aube \\
\hline
\end{tabular}

\subsection{Nombres de barcos}

Aunque los nombres de los barcos se han traducido literalmente en la versión española, en la versión francesa observamos ligeros cambios.

\begin{tabular}{|l|l|l|}
\hline INGLÉS & ESPAÑOL & FRANCÉS \\
\hline The Storm Dancer & La Danzarina de las Tormentas & La Cavalière des Tornades \\
\hline The Wind Witch & El bruja del Viento & La Charmeuse du Vent \\
\hline
\end{tabular}


En el primer ejemplo, "The Storm dancer" pasa en francés a ser "la jinete de los tornados" y en el segundo caso el término "witch" sufre una ligera modulación y hace que el barco pase a denominarse "la encantadora del viento".

\subsection{Nombres de idiomas}

En la novela analizada tan solo encontramos una referencia a un idioma (en el resto de la saga aparecen más). Se trata de "The Common Tongue", la lengua que se habla en los siete reinos. Como podemos ver en la tabla, la versión española ha optado por una traducción literal, mientras que la versión francesa recurre a la modulación y se refiere a ella como "la langue classique" (la lengua clásica/tradicional).

\begin{tabular}{|l|l|l|}
\hline ORIGINAL & ESPAÑOL & FRANCÉS \\
\hline The Common Tongue & La lengua común & La langue classique \\
\hline
\end{tabular}

\subsection{Nombres de animales}

En cuanto a los nombres de animales, vemos que la tendencia tanto en español como en francés es traducir los nombres que tienen algún tipo de connotación semántica y dejar igual que en el original los que no aportan ningún tipo de información al lector.

\begin{tabular}{|l|l|l|}
\hline ORIGINAL & ESPAÑOL & FRANCÉS \\
\hline Ghost & Fantasma & Fantôme \\
\hline Nymeria & Nymeria & Nymeria \\
\hline Lady & Dama & Lady \\
\hline Grey Wind & Viento Gris & Vent Gris \\
\hline Shaggydog & Peludo & Broussaille \\
\hline Balerion & Balerion & Balerion \\
\hline Meraxes & Meraxes & Meraxès \\
\hline Vhagar & Vhagar & Vhagar \\
\hline Summer & Verano & Eté \\
\hline Whitey & Blanca & Blanchoł \\
\hline Dancer & Bailarina & Danseuse \\
\hline
\end{tabular}

\subsection{Nombres de espadas}

Dada la temática medieval en la que se enmarca la obra y que muchos de sus personajes son expertos guerreros es habitual que estos pongan un nombre a sus espadas. En la tabla siguiente recogemos algunos ejemplos:

\begin{tabular}{|l|l|l|}
\hline INGLÉS & ESPAÑOL & FRANCÉS \\
\hline Ice & Hielo & Glace \\
\hline
\end{tabular}




\begin{tabular}{|l|l|l|}
\hline Needle & Aguja & Aiguille \\
\hline Lion's Tooth & Colmillo de León & Dent-de-Lion \\
\hline Heartsbane & Veneno de Corazón & Corvenin \\
\hline Dawn & Albor & Aube \\
\hline
\end{tabular}

Como se puede observar, la tendencia de ambos idiomas es traducir literalmente los nombres, aunque también se detectan otras técnicas de traducción en casos puntuales. Por ejemplo, la espada denominada en la obra original Lion's Tooth se traduce literalmente al francés, pero en la versión española se opta por la particularización al renombrarla "Colmillo de León", pues al hacer referencia a un animal dicho término encaja mejor.

Con Heartsbane observamos que se efectúa una traducción literal al español y para el francés se recurre a la creación de un neologismo a partir de la palabra "cor" que en occitano significa "corazón" y la palabra "venin" (veneno).

\section{Conclusión}

En el presente artículo hemos llevado a cabo el análisis de la primera novela de la saga Canción de hielo y fuego con tres objetivos principales: establecer una clasificación de los nombres propios incluidos en ella, comprobar las técnicas de traducción de nombres propios más habituales en español y francés y comparar ambas versiones meta para averiguar si los procedimientos de traducción eran los mismos en las dos lenguas.

En cuanto al primer objetivo dividimos nuestro corpus en ocho apartados, en función de los nombres que íbamos encontrando. Cabe aquí destacar que para un futuro análisis más exclusivo en el que el corpus esté compuesto por todas las novelas de la saga, sería necesario ampliar el número de categorías en función de los nuevos nombres que vayan surgiendo, por ejemplo, nombres de espadas.

Una vez hecha la clasificación pasamos a analizar las técnicas de traducción por separado en las dos versiones. Observamos que la novela contiene una gran cantidad de nombres propios que aportan información al lector, no obstante muchas veces en las traducciones esta se pierde. Comprobamos que, si bien, en muchos casos las dos traducciones coinciden en la estrategia de traducción empleada, el francés tiende mucho más a trascribir los nombres propios, aunque estos tengan algún tipo de connotación semántica. En muchos de estos casos se requería una traducción creativa (por ejemplo, el caso de Winterfell, Raventree o The Eyrie), sin embargo las técnicas de traducción empleadas en francés y español son muy diferentes. Mientras que el español opta ante estos problemas recurrir a la creación discursiva, el francés mantiene los mismos nombres que en inglés, con la consecuente pérdida de información para el lector meta. Asimismo, en los casos en los que el traductor se ha visto obligado a realizar una traducción creativa, comprobamos que la versión francesa recurre a palabras del occitano a partir de las cuales crea los neologismos pertinentes, si bien el número de ejemplos es reducido, por lo que no podemos confirmar que esta sea la tendencia general.

En el siguiente gráfico resumimos las técnicas de traducción empleadas para traducir los nombres propios. 


\section{Resumen de las técnicas de traducción de nombres propios}

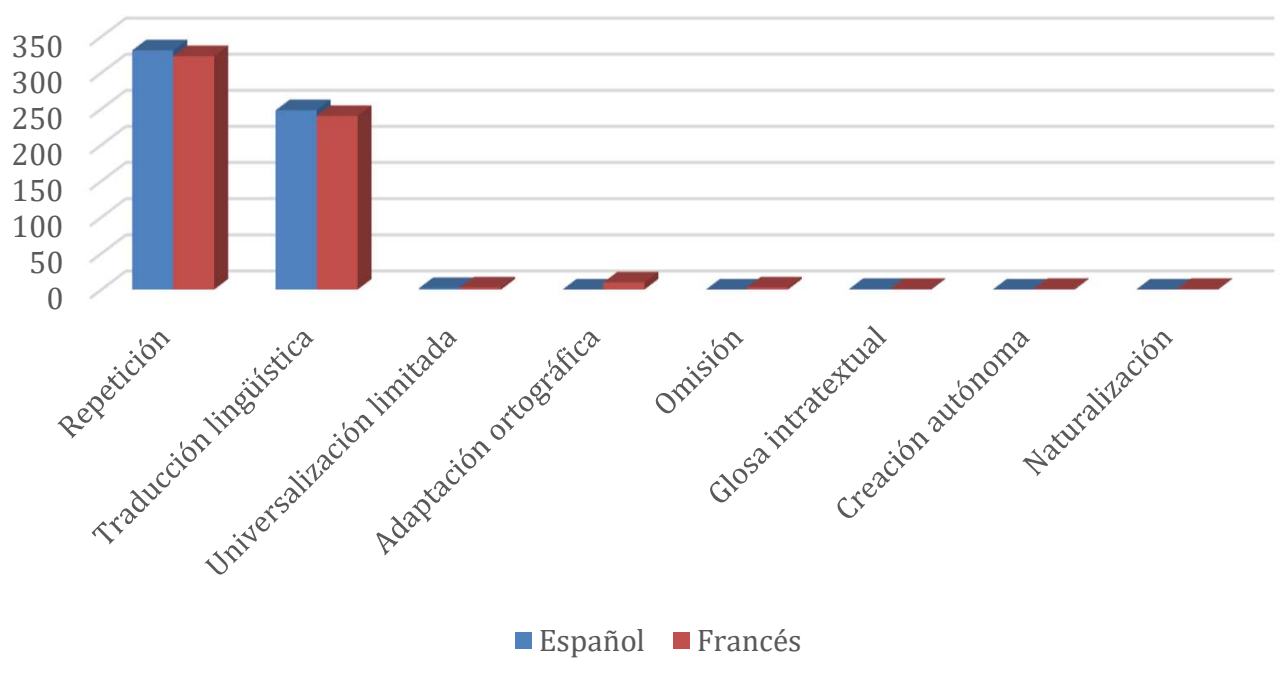

Como se puede observar, de acuerdo con la tipología propuesta por Franco (1996) existe una clara tendencia al uso de técnicas de conservación, entre las que destacan la repetición y la traducción lingüística. Esto es debido a que una gran parte de los nombres propios que aparecen son antropónimos referenciales que no aportan ningún tipo de información sobre el personaje y muchos de ellos se corresponden con personajes secundarios. Solo el francés decide adaptar de vez en cuando el nombre a la ortografía meta, pero aun así no es la tónica habitual. Para los casos en los que el nombre contiene información semántica, se observa una mayor tendencia a la traducción lingüística en ambas lenguas.

En el caso de los topónimos observamos que la tendencia es justo la inversa, pues la gran mayoría aportan información sobre las características del lugar. Para solventar esta dificultad ambas lenguas tienden a utilizar la traducción lingüística, si bien en el caso de francés son más numerosos los casos en los que el uso de la repetición ha privado de información al público meta (Winterfell, Eyrié, The Neck). Al tratarse de una obra enmarcada en el género fantástico, comprobamos que una gran parte de los nombres de lugares son puramente referenciales y se corresponden con lugares extranjeros, por lo que en estos casos ambas lenguas meta siguen optando por la conversación, pero recurren más a la repetición (Myr, Norvos, Sherrer, Aggo, Rakkaro, Tyrosh, Qohor).

Por último, comprobamos que aunque esta novela contiene un número elevado de nombres propios, al enmarcarse dentro del género fantástico, los traductores no siempre podrán recurrir al uso de la técnica de la repetición, sino que será necesario buscar otras técnicas que permitan conservar la carga semántica del nombre en la lengua meta. Asimismo, cabe aquí destacar que la novela objeto de estudio pertenece a una saga, por lo tanto, todas las decisiones de traducción que se tomen en la primera novela condicionarán inevitablemente la labor de los traductores de las siguientes partes de la saga. 


\section{Bibliografía}

AINAUD, J., A. ESPUNYA \& D. PUJOL (2003): Manual de traducció anglès-català. Vic: Eumo Editorial.

CUÉLLAR, C. (2014): "Los nombres propios y su tratamiento en traducción". Meta, 592, pp. 360-379.

FRANCO, J. (1996): Condicionantes de traducción y su aplicación a los nombres propios (inglés-español). Tesis doctoral. Universidad de Alicante. Disponible en línea:

<https://rua.ua.es/dspace/bitstream/10045/3508/1/Franco\%20Aixelá,\%20Javier. pdf>

FUNDÉU (2007): Traducir (o no) los topónimos. Disponible en línea: <https://www.fundeu.es/noticia/traducir-o-no-los-toponimos-3617/>

GARCÍA YEBRA, V. (1983): En torno a la traducción. Madrid: Gredos.

LODGE, D. (1992): The Art of Fiction. London: Penguin Books.

MARTIN, G. R. R. (1996): A song of Ice and Fire. A Game of Thrones. New York: Bantam Spectra.

MARTIN, G. R. R. (2002): Canción de hielo y fuego 1. Juego de tronos. C. MACíA (trad.). Barcelona: Gigamesh.

MARTIN, G.R.R. (1999) : Le Trône de fer, tome 1: La Glace et le Feu. Paris: Pymalion Editions.

MOYA, V. (1993): "Nombres propios: su traducción". Revista de Filología de la Universidad de La Laguna, 12 (1993), pp. 233-247.

NEWMARK, P. (1988): A Textbook of Translation. Nueva York: Phoenix Elt.

NEWMARK, P. (1992): Manual de traducción. Madrid: Cátedra.

RAMOS, A. (1999): "Teoría y práctica de la traducción literaria". Mirandum, 8, pp.1-2.

REAL ACADEMIA ESPAÑOLA. (2001): Diccionario de la lengua española (22." ed.). Disponible en línea: <http://www.rae.es/rae.html> 